\title{
Entre la pedagogía de la ausencia y la hospitalidad. Reflexiones en torno a las diversidades y ser docente en Chile
}

\author{
Between the pedagogy of absence and hospitality. Reflections \\ on diversities and being a teacher in Chile
}

\section{Mauricio Vergara Rodríguez*}

\begin{abstract}
RESUMEN
El presente artículo busca reflexionar sobre el significado de las diversidades, desde la lectura de Carlos Skliar y el primer Martín Heidegger, como una búsqueda para encontrarnos, convivir y coexistir en los espacios educativos. A partir de la propuesta de Carlos Skliar, se comprende la escuela y la formación docente desde una pedagogía de la ausencia y una pedagogía de la hospitalidad. Ambas generarían un correlato en las formas de apropiación del ser docente desde la ausencia y la hospitalidad que se pueden proyectar a los procesos de formación inicial docente. Establecer la ausencia significa comprender al otro como un ser incompleto, que debe ser para un mañana y que aún no es. La hospitalidad, en cambio, es una invitación al otro desde el respeto y la tolerancia, pero siendo entendido como anormal y que también debe ser mejorado. Al finalizar el artículo se invita a la búsqueda de una pedagogía de la apertura, comprensión y común-acción para abordar las diversidades como un co-habitar, considerando que las diversidades se establecen en nuestras gramáticas como formas de comprender el mundo y escuchar de manera ética a los otros. Se plantea el término "diversidades" en el sentido de ampliar el concepto y no como una forma de pensar unívoca.
\end{abstract} Palabras clave:

diversidades, pedagogías, formación docente inicial, formas de comprensión del mundo. 


\section{SUMMARY}

This article seeks to consider the meaning of diversities, from the reading of Carlos Skliar and the first Martin Heidegger, as a search to meet, coexist, and coexist in educational spaces. Based on Carlos Skliar's proposal, school and teacher training are understood from a pedagogy of absence and hospitality. Both would generate a correlation in the forms of appropriation of being a teacher from absence and hospitality that can be projected to the processes of initial teacher training. To establish absence means understanding the other as an incomplete being, who they must be for tomorrow and who they are not yet. Instead, hospitality is an invitation coming from respect and tolerance, but to be understood as abnormal and needing improvement. We invite the reader to search for a pedagogy of openness, understanding, and joint action. Also, to approach diversities as a co-inhabitation, considering they are established in our syntaxes as ways of understanding the world and listening ethically to others. We use the term "diversities" to broaden the concept and not as a univocal way of thinking.
Key words: diversities, pedagogies, initial teacher training, ways of understanding the world. 


\section{Introducción}

"Una de las afirmaciones más memorables que recuerdo de mi formación en educación especial quizá fuera la del criminólogo estadounidense Ken Polk, cuando dijo que, para muchos niños, la experiencia de la escuela es la experiencia cotidiana de la humillación y el dolor."

(Roger SLEE, 2012, P. 29)

El presente artículo es una reflexión a partir de la pregunta: ¿qué significa ser docente en el contexto actual de formación docente inicial en Chile? El espacio que puede preparar y orientar el marco para responder la pregunta está en las diversidades e inclusión, porque, en primer lugar, cómo comprendemos las diversidades se vincula con las formas en que comprendemos el mundo que habitamos (Druker, 2020) y, más importante aún, "la forma de escolarización que construirá ese mundo" (Slee, 2012, p. 32). Por ello, las formas de comprender las diversidades permiten que se desplieguen diferentes maneras de ser profesor(a). En segundo lugar, porque en la medida que revisamos cómo se han comprendido las diversidades e inclusión en el sistema educativo podemos vislumbrar que se orientan hacia una pedagogía de lo que no es aún (ausencias) o una pedagogía de la hospitalidad (Skliar, 2002). Por lo mismo, se escolariza para estar en un futuro que no es aún o para una experiencia que invita a la uniformidad.

Así, las diversidades como búsqueda permitiría salirnos del análisis técnico y aproximarnos desde la aparición en la experiencia escolar de un yo y un tú, de un yo y un otro en comunidad, situación que no ha sido la característica del sistema educativo (Slee, 2012). Además, asumir la diversidad como diversidades significa considerar múltiples aportes desde diferentes epistemologías y filosofías (Ocampo, 2018; Druker, 2020) que nos lleven otra vez a reflexionar sobre las relaciones éticas (Skliar, 2016). De hecho, lo señala Roger Slee (2012):

La educación inclusiva nos invita a pensar sobre la naturaleza del mundo en que vivimos, el mundo que preferimos y nuestro papel en la configuración de ambos mundos. A partir de ahí, siguen cuestiones técnicas sobre la investigación, los recursos, el curriculum, la pedagogía, la evaluación y las ecologías de las escuelas y el desarrollo humano. (p.32) 
Si esto es así, vale decir, que las diversidades orientan procesos pedagógicos, esto podría ocurrir de acuerdo con Carlos Skliar (2002) en dos sentidos: ausencia u hospitalidad. El docente que actúa en la escuela enmarcado por contextos, paradigmas, experiencias personales y relaciones sociales que se establecen en la interacción pedagógica, se ve también comprendido desde la ausencia y la hospitalidad. En otras palabras, podríamos aventurar que, si las diversidades se despliegan en la pedagogía de la ausencia, el profesor se apropia desde la ausencia $\mathrm{y}$, si las diversidades se despliegan en la pedagogía de la hospitalidad, el profesor se apropia desde la hospitalidad.

Ahora bien, intentaremos reflexionar con estas ideas iniciales para abrir otro espacio: establecer un diálogo entre los profesores y las diversidades con aspectos de la filosofía de Carlos Skliar (2002) y del primer Martín Heidegger (1919-1927). Este diálogo permitiría la emergencia del convivir y coexistir frente a los desafíos de las diversidades. En ese sentido, se abriría una pedagogía del cohabitar, una pedagogía de estar-siendo-en-el-mundo. No una pedagogía de las referencias externas de un mundo o realidad sobre otra, sino del diálogo y del encuentro (Restrepo-Pérez, Restrepo-Pérez y Jaramillo-Ocampo, 2017). Al encontrarnos y conversar puede emerger la política y la ética, ambas claves para el proyecto ya no de una educación diversa, sino inclusiva (Slee, 2012; Skliar, 2016).

Esta búsqueda de unir y hacer dialogar diferentes puntos de vistas (representados en campos semánticos diferentes, tal como se pueden comprender las lecturas desde los ámbitos educativos y filosóficos) puede mostrar que, lo que ha olvidado la educación del siglo XX es la vida. La comprensión de la educación está estrechamente ligada a la vida y, más que definir cierto tipo de saber y hacer, la educación se relaciona con el ser y el estar siendo (Quay, 2013). En cierta medida, reflexionar sobre las diversidades requiere volver a conectarse con la vida. $Y$, respecto de la vida, los docentes - y quizás también las escuelas- se han extraviado.

En primer lugar se presentarán las tensiones del proceso de inclusión en las escuelas en Chile, considerando algunas prácticas pedagógicas que dan cierto sentido a cómo se comprenden las diversidades e inclusión. En segundo, las tensiones de la formación docente en las últimas décadas en Chile. Ambas se consideran a 
partir de las ideas de Carlos Skliar (2002). En tercer lugar se describirán algunos aspectos iniciales de la propuesta del dasein de Heidegger (1997), como forma de volver a describir los aspectos que pueden enmarcar la reflexión sobre las diversidades. Finalmente, se establecen consideraciones como una forma de seguir debatiendo y reflexionando.

\section{La pedagogía de la ausencia y la hospitalidad en las escuelas}

Algo en la escuela no cuaja, no va bien. Y no es solo por los estudios asociados a la efectividad en los aprendizajes de sus estudiantes y las diferencias en la calidad de la educación en Chile. Lo que no va bien es la herencia de entender la escuela desde el sentido de lo dado y aquello por completar o incompleto (Skliar, 2005). Lo dado significa aquello que hay que conocer, aprender, y que lo por aprender está fuera de nosotros, organizado en disciplinas, métodos y formas de evaluación, expresado en "prácticas centradas en la cobertura curricular y transmisión de contenidos" (Osandón, et al., 2018, p. 29). Y el sentido de lo incompleto, o aquello que está por completar, entiende que los participantes entran a ese espacio escolar para ser completados por otros desde ideas preconcebidas, comparados con lo que deberían ser, "entendiéndose la alteridad como deficiente" (Skliar, 2005, p. 13). Podríamos resumir lo que significa esta relación que emerge en la escuela a partir de lo dado y lo incompleto como:

La invención -y la construcción y la producción- de la incapacidad del otro es aquello que posibilita el nacimiento de la figura del explica-dor. Y justamente es el maestro, la maestra, el explicador que ha inventado al incapaz para justificar su explicación. Por tanto, el explicador y el incapaz constituyen un binomio inseparable de todas las presuposiciones argumentativas de la pedagogía, actuales y pasadas. No hay maestro explicador sin alumno incapaz previamente construido. Y en la misma medida en que el maestro torna más amplia la magnitud de su explicación, el cuerpo del alumno se va haciendo cada vez menor: es empequeñecido por la explicación. Así, la explicación es un constante y perverso proceso de empequeñecimiento del otro o, en las palabras de Ranciére, de embrutecimiento del otro (Skliar, 2005, p. 14). 
Las prácticas pedagógicas se basan en el principio de lo incompleto y razón explicativa. En ese sentido, esconde o naturaliza las alteridades deficientes. Las prácticas pedagógicas necesitan de otro, llamado niña o niña, definiendo para ellos de manera predeterminada qué es aprender, qué aprender, cómo aprender y para qué aprender. El niño o niña debe aprender aquello por lo cual no ha preguntado, reduciéndose a un "sujeto pasivo, que ha de ser rellenado de contenidos" (Recalcati, 2016 , p. 25). En la escuela siempre se aprende para un mañana que nos permitiría entender qué somos y cómo deberíamos actuar. El mañana es lo por venir y, ciertamente, en el presente no está. Aprendemos, entonces, en función de lo que no está, y la pedagogía de los contenidos se convierte en una pedagogía de las ausencias. Esto quiere decir que nos relacionamos suponiendo al otro, pero ese otro aún no es y esperamos que sea algo configurado "sobre dos principios «pedagógicos» tan austeros como inexpugnables: (a) está mal ser aquello que se es y/o se está siendo; (b) está bien ser aquello que no es, que no está siendo y que nunca se podrá-o querrá-ser" (Skliar, 2002, p. 147).

Si se revisan las indicaciones de los documentos ministeriales chilenos para incorporar la diversidad e inclusión, se evidencia una cierta pedagogía de la hospitalidad: invitar al otro a que sea desde la tolerancia y el respeto, reconociendo ciertas condiciones de exclusión que la escuela debe mejorar para crear una sociedad más justa y equitativa (Parra et al., 2020), replicando el modelo de la educación especial para la escuela regular (Manghi et al., 2020), "trasladando la idea de déficit asociado a la diferencia a otras identidades presentes en la sala de clase. De esta manera se reinstala la idea de que lo diferente es anormal y que debe ser mejorado" (Matus \& Rojas, 2015, p. 50). Por lo mismo, existen dos pedagogías conviviendo: la que supone a otro que no está, por ejemplo, en el entramado curricular, y otra pedagogía de la hospitalidad, que invita al otro a participar, pero que, al ser invitado a participar, debe ser mejorado.

En ese sentido, las orientaciones para incluir lo diverso tienden a insistir en "la binarización de lo humano, en tanto se constituirían dos tipos de alumnos: el normal y el con NEE" (Parra et al., 2020, p. 17), considerando un valor como hegemónico, el cual articula las relación de inclusión y exclusión y el rol rector o normalizador de algunas identidades sobre otras (Mattus et al., 2019). Por ejemplo, "cada vez 
que un profesor quiere imaginarse que su sala de clase es homogénea, cualquier tipo de sujeto que venga a interrumpir esa homogeneidad será pensado como un problema" (Matus \& Rojas, 2015, p. 54), y sentirá temor sobre cómo actuar ante el otro, qué decir y qué no decir, qué hacer y cómo relacionarse con lo que debe ser (normal) y aquello que no actúa y no es como esperamos (anormal). De hecho, cuando un estudiante no responde a lo que esperamos de él, lo invitamos a unirse a la regla, a estar sentado, motivado, escuchando. Esperamos que lo que tenga que suceder, suceda adecuándose a lo normal.

Las relaciones entre lo normal y anormal son relaciones de poder (Sverdlick, 2019), se configuran desde dispositivos de poder. La escuela misma, al suponer un tiempo y un espacio unívocos establece una lógica de la mismidad y expulsa - catalogando de "diferentes", "con dificultades" - a aquellos que tienen otros tiempos, otros espacios y otras formas de aprender. O como señala Baker (2002):

...un modo de aprender establecido se puede convertir en la discapacidad de aprender, a la luz de la impaciencia y la estructura de la escuela que presenta un número limitado de formas, con expectativas rígidas sobre lo que significa un desempeño oportuno. (p. 685; traducción propia)

Por lo mismo, la escuela crearía la anormalidad, y la discusión sobre la normalidad y anormalidad no podría situarse en el individuo, sino que:

en los procesos históricos, culturales, sociales y económicos que regulan y controlan el modo a través del cual son pensados e inventados los cuerpos, las mentes, el lenguaje, la sexualidad de los otros. Para expresarlo aún más contundentemente: las deficiencia no es una cuestión biológica sino una retórica cultural. (Skliar, 2002, p. 118)

En el sistema educativo en Chile aún no emerge un sentido y una práctica en los que se reconozca y articule una propuesta en la cual las referencias a la identidad y a la diferencia se asuman desde una perspectiva del poder que se ejerce en el curriculum, ni del tipo de identidades que el docente tiene respecto de su propio ejercicio (Mattus et al., 2019). De acuerdo con Verónica López y colaboradores (2018), que investigan sobre las posibilidades de una educación inclusiva, existen tres nudos críticos que tensionan las propuestas de cambios que se 
han realizado en el último tiempo en el sistema educativo chileno: tensiones conceptuales o ciertas incoherencias entre las lógicas de comprensión de los nuevos fenómenos educativos (como la inclusión, por ejemplo); el modelo de mercado y lo que llaman una nueva "arquitectura educacional", basada en la rendición de cuentas e incentivos individuales, con lógicas homogeneizadoras y que castigan las diferencias.

Si bien estas nuevas políticas apuestan por un proceso de crítica, autorreflexión y retroalimentación de la comunidad educativa, para la elaboración de diferentes proyectos educativos (inclusivos), las cuestiones sobre las relaciones de poder, como productoras de las identidades y diferencias (Da Silva, 2014), quedan invisibilizadas. Vale decir, se propone como un tema ya dado y no como una cuestión a producir, es decir, proceso en el que preguntar sobre “ $¿$ Cómo la identidad y diferencia son producidas? ¿Cuáles son los mecanismos y las instituciones que están activamente envueltos en la creación de la identidad y su fijación?" (Id., p. 99) sea una cuestión relevante.

La escuela ha sido el espacio educativo configurado como hegemónico en los Estados nacionales, para transmitir saberes culturales que aseguren la integración de los individuos a la sociedad (Pineau, 2001). Con el avance de las reformas neoliberales, la escuela ha puesto el éxito de la transmisión de esos saberes en políticas de rendición de cuentas públicas (Parcerisa y Falabella, 2017) y currículos organizados para verificar y medir el logro de los objetivos de aprendizaje (Slee, 2012). La necesidad de reflexionar sobre nuevas formas de sentir, pensar y ser en la sociedad, y particularmente los temas asociados a las diversidades, significa tensionar o reflexionar sobre las prácticas de escolarización y ser conscientes de los tipos de pedagogía que estamos practicando.

Algunos autores han señalado, desde la perspectiva del currículo, que la escolarización supone establecer espacios en los que las personas aprendan determinadas experiencias, definiendo un tipo de aprendizaje en similares tiempos y espacios (Parra et al., 2020), y dejando de lado otras experiencias. La educación, y particularmente la escuela, significa ciertas narrativas sobre el conocimiento, y esas narrativas suponen y fijan un tipo de sujeto. Aún estamos pensando la escuela desde la perspectiva técnica y racionalizada de Tyler: la escuela normaliza (Tadeu, 2014), comprendemos al otro como nosotros mismos. La comprensión del otro como yo mismo solo deja la posibilidad 
de comprender las diversidades como un efecto de relaciones entre un grupo que establece lo deseable y otro que se debe relacionar y aprehender desde esa relación ${ }^{2}$. Y esa relación aparece en la práctica pedagógica como una pedagogía de la ausencia y de la hospitalidad. Es más, la escuela es la expresión de cierta pedagogía de las ausencias: no emerge ninguna relación entre un yo y un tú.

\section{La formación docente en Chile: una forma de reproducir la pedagogía de la ausencia (técnica) y de la hospitalidad}

En las políticas de formación docente en Chile, los aspectos referidos a la diversidad han sido incluidos como programas de formación docente configurados desde una antropología neoliberal (Firtz, 2016) y una racionalidad técnica (Grundy, 1991), basadas en el logro de competencias (CNA, 2018), además de una pedagogía que, muchas veces, está desconectada de la realidad escolar (Garay \& Sillar, 2018); una organización estructural del conocimiento disciplinar y pedagógico (Cherryholmes, 1999), y una fuerte instalación de procesos de acreditación y rendición de cuentas (Biesta, 2014).

Frecuentes debates en Chile han señalado la gran dificultad que presenta el curriculum formativo de profesores. Según diversos autores, la formación docente tiene una desvinculación con la realidad escolar y, sobre todo, logra resultados muy bajos en el desarrollo de competencias reflexivas (Manzi, 2011; Montecinos, 2018; Vaillant, 2010, Mineduc, 2018). Tal como señalan Labra y Fuentealba (2011), en la formación inicial "no se reconoce la multidimensionalidad de los fenómenos educativos ni la posibilidad de que los profesores sean generadores de conocimiento al reflexionar sistemáticamente en y acerca de su quehacer docente" (p. 145). O, como releva Tardif (2004), se estaría desarrollando un modelo de formación aplicacionista, en el que los futuros profesores cursan un sinfín de materias que luego deben

2 Solo como un ejemplo, en el currículo de Primero Básico, en Chile, en la asignatura de Música se debe aprender diferentes tradiciones culturales para conocer ritmos, métricas y características musicales, entre ellas se destaca "el negro Cirilo" como representante de la música infantil y tradiciones latinoamericanas. El programa presenta al negro Cirilo sin posibilidad de discusión sobre cómo compremos al otro desde los aspectos musicales (Ministerio de Educación, 2013, p. 40). 
aplicar en las prácticas, considerando estos saberes como productos de otras disciplinas y no precisamente a partir de sus experiencias.

De acuerdo con esto, las políticas públicas se han confeccionado desde formas de pensar estructurales (Cherryholmes, 1999) y se han propuesto soluciones desde lógicas de innovación y gestión de la práctica docente, buscando generar relaciones más eficaces para el ejercicio de la docencia (De Gregorio y Bruns, 2016; Ávalos 2013). En este escenario, se han generado prácticas que naturalizan estrategias, materiales y categorías (Montecinos, 2016) para significar las relaciones en el aula, sin mayor conexión con las creencias, valores, significados e ideologías de los docentes (Bartolomé, 2008), configurando al profesor como un sujeto técnico, aséptico, despolitizado y sin historia personal (Freire, 2016). Otras investigaciones señalan que, en el contexto de las políticas de orden neoliberal, se estaría configurando una subjetividad docente tensionada por enmarcarse dentro de las categorías de desarrollo profesional docente (Fardela y Sisto, 2015), así como otras que, durante los últimos años, se han enfocado en los significados, creencias, relaciones y percepciones de los docentes sobre lo que hacen (Guerra y Balmaceda, 2015), y diferentes formas de representaciones que tienen los estudiantes en formación inicial (Aranda, 2011).

Es necesario detenerse en un punto muy relevante a propósito de las diversidades: la desconexión entre las propuestas sobre la formación docente inicial con las creencias, valores y significados que llevan consigo las personas. El profesor en formación no puede verse a sí mismo desde la propuesta originada por el Ministerio de Educación de Chile, ya que no se puede desplegar desde esta configuración del docente ya dada, y se eclipsa la opción de convertirse en un docente comprendido como una apertura al mundo, al lenguaje y al convivir. El docente tiene que ser para otro, y ese otro lo valida en tanto representa un saber y un saber-hacer no originados desde su propios saberes, experiencias y mundo. Los mismos procesos de formación docente replican las pedagogías de la ausencia y la hospitalidad. Le decimos al profesor en formación inicial que es lo que debería hacer y saber, o, adaptándonos a ellos, lo invitamos a responder a la norma: que domine una disciplina, reflexione en la práctica y sepa diagnosticar a otro para su aprendizaje. Esto último, tal como señalan Claudia Matus y Ca- 
rolina Rojas (2015), es parte del ejercicio docente: saber diferenciar el aprendizaje de otro a partir de una norma.

Por lo mismo, la formación docente en Chile ha olvidado que los profesores son personas que actúan en un campo de complejidad, en el cual actúan dimensiones históricas, culturales, sociales y personales. El profesor también ha sido mirado como el otro entendido como anormal: sin historia, sin cuerpo, sin sexualidad, género, edad, clase social, etcétera (Skliar, 2002). Ciertamente, se ha olvidado que los profesores poseen una historia vital, vale decir, una identidad que no es solo profesional, que se vincula con un estar-sentir-emocionar propios (Naranjo, 2014; Casassus, 2006). Al respecto, Romanowski (1997) señala que la historia personal, las creencias y el contexto escolar explican lo que un profesor realiza en sus clases, mientras que Tardif (2004) agrega que lo que hace un docente se relaciona con ciertas experiencias significativas en su vida personal y profesional. La formación inicial docente en Chile está invisibilizando la identidad y el proceso de ser personas de los docentes. Dicho de otro modo, se han formado docentes sin considerar los puntos de vista de los propios docentes, más allá de propuestas técnicas e instrumentales (Muñoz, 2011). Se está formando al docente en la misma postura en que la diversidad llega a la escuela: desde un deber ser, para un mañana que todavía no es. Una formación docente desde la pedagogía de la ausencia.

De hecho, si se quisiera resumir la propuesta formativa de docentes en Chile, se podría coincidir con Grundy (1991), respecto de que responde a una racionalidad claramente técnica. Esto quiere decir que una de las principales características del proceso formativo es la reproducción y, utilizando un esquema a partir de la lectura de la Ética a Nicómaco de Aristóteles, la racionalidad técnica es la reproducción de un eidos inicial. Entonces, ¿cuál es la idea inicial que el profesor debería reproducir?

En primer lugar, se sigue pensando al docente como transmisor de aprendizajes, desde una propuesta que se puede relacionar con la razón pedagógica basada en la explicación (Ranciére, 2007; Osandón et al., 2018), en la cual uno de esos aspectos es el intercambio respetuoso de diferentes identidades y manifestaciones culturales. En segundo lugar, existe una predeterminación de qué es lo deseable respecto a cómo abordar las diversidades (Manghi et al., 2020). No se expresa 
como un proyecto a construir, sino más bien como una particular forma de ser, con perspectivas de incluirse en estilos de vida ya entendidos como sostenibles y buenos.

\section{Una aproximación desde Martín Heidegger: reflexionando sobre el ser docente y las diversidades a partir de algunas ideas del dasein}

Desde mi punto de vista, la filosofía de Heidegger es fundamental para romper estas maneras de pensar y que se logre abrir nuevas perspectivas, puesto que con él se reconoce que toda forma de relación con los otros se basa en que estamos abiertos a comprender, a que convivimos y coexistimos de una forma particular y originada en el ser-estar-en-elmundo. Sobre todo porque el pensar de Heidegger, que en sus primeros años está estrechamente relacionado con el ser-estar-en-el mundo constitutivo del dasein, se puede comprender como una posible lectura de lo que significan esos otros modos de poder ser que implican la aparición de los otros.

En ese sentido, la pregunta sobre las diversidades nos lleva a reconocer la vida y el ser humano como articuladores de las relaciones de poder, sociales y culturales. La crítica de Heidegger a la metafísica puede ser una forma de orientar la búsqueda. Esta crítica, según Wolfgang Welsh (2008), corresponde a que las referencias hacia qué es el hombre y al tipo de conocimiento que se válida y en el cual se dan las relaciones de poder, deben ser hechas desde el ser. Es una propuesta ontológica, en la cual la referencia al ser como articulador del ser humano y sus prácticas es relevante. Por lo mismo, el espacio que se abre para la reflexión se relaciona con el "ser docente-ser otro", y eso nos posiciona desde un punto de vista distinto del de los procesos de formación estandarizados, propuestos desde miradas ajenas, porque "comprender al hombre a la luz de su relación con el ser" (Welsh, 2008, p. 96) significa preguntarse por las condiciones que permiten el convivir y coexistir.

Heidegger aclara algunas ideas sobre las que estamos reflexionando en su obra Ser y Tiempo, particularmente en los parágrafos 12 y 13, destacados por Jorge Acevedo (1999), y, según Luis Uribe Miranda (2002), en los parágrafos 31, 32 y 34 se expone el marco el que iniciar la reflexión con el filósofo, sobre la constitución de sus ideas fundamentales y sus posteriores búsquedas. 
En primer lugar, el dasein está-en-el-mundo, en apertura y comprensión. Solo después se da una relación epistémica. Esto quiere decir que el encuentro con el otro ocurre como una proyección de nuestra propia existencia, pero no como ocultamiento, sino como copertenencia. Un tema importante que Heidegger permite dilucidar, puesto que lo que está en juego no es la racionalidad solamente expresada en sus reglas, sus fines, y en el cómo se conciben las diversidades, sino que en una forma de ser y de habitar el mundo, de comprender las relaciones sociales, al ser humano, la naturaleza, etcétera.

En segundo lugar, el dasein está constituido originariamente por el comprender. De hecho, lo considera un modo fundamental del ser del dasein y no debe confundirse con la tensión entre explicación y comprensión. Esta tensión debería ser interpretada como una derivación de este comprender primario, originario del dasein. La comprensión originaria a la que se refiere Heidegger quiere decir que, siendo el $d a-$ sein ser ahí, y el "ahí" estar en el mundo, lo que se da en la comprensión es la apertura y significatividad que tiene el dasein con ese mundo.

El estar-en-el-mundo y el dasein son apertura significativa, el mundo está abierto como tal en un comprender. Que el dasein sea comprensión -originaria y no como tradición del conocimiento- significa que se lanza, se arroja a las posibilidades de su poder ser, de sus posibles determinaciones. Comprender es siempre comprender en torno a posibilidades, estando en un mundo, dispuesto de manera afectiva y en apertura hacia..., como un horizonte indeterminado. No hay algo que ya esté dado.

Ahora bien, existe un aspecto muy relevante para destacar la lectura de Heidegger, al tenor de la reflexión sobre las diversidades, y es que estas formas de comprensión "están determinadas por el discurso" (Heidegger, 1997, p. 160). Vale decir, es el lenguaje el que pone al ser humano en contacto "con el ser, la verdad, con el mundo y la ética" (Uribe, 2002, p. 45). El lenguaje se constituye como el espacio en que emerge la relación entre los seres humanos, pero no con el sentido de una competencia lingüística, en la cual podamos comunicarnos entre todos — como si todos pudiésemos hablar el mismo idioma-, sino que en el lenguaje emerge un mundo con ciertos órdenes, formas de conocer, jerarquías, valores, creencias y ecosistemas (Masolo, 2014). Habitamos en el lenguaje y, a partir de él, podemos tomar algo en re- 
lación con el otro. Es decir, estamos con el otro a partir de lo común, por la comunicación, y, en ella, podemos ser nosotros y ser con otros.

Es importante decir que lo común no supone la mismidad, no supone la homogeneización. Lo común supone un punto de partida en el sentido de común-acción, en el cómo nos interrelacionamos, desde dónde emergemos como seres humanos: lenguaje-interpretación, cuerpo y emociones. La pedagogía de las ausencias en la escuela y en la formación docente inicial caducaría en la medida en que la práctica pedagógica ya no pregunta sobre qué, cómo y para qué aprenden los otros, sino cómo interpretamos el compartir los diferentes mundos que somos. En ese sentido, el problema de las diversidades no sería cómo lograr que todos aprendan sin exclusiones los mismos objetivos, llevándolo a la dificultad curricular y didáctica, sino que aprender el lenguaje de la responsabilidad (Han, 2017), del interpretar los mundos que nos constituyen. Por lo mismo, las diversidades se relacionarían más con aprender a callar y aprender a escuchar, como una forma de ser conscientes de las gramáticas o mundos que nos constituyen, el cuerpo desde donde emerge lo que escuchamos y sentimos (Duschats$\mathrm{ky}, 2017)$ y las relaciones sociales que se establecen en un contexto histórico particular.

Las diversidades serían la emergencia de diferentes mundos y sus formas de comprenderlos. No se vinculan con las características propias de un sujeto, sino con una forma de interacción entre personas. Es cierto, no podemos ser el otro. Y eso es maravilloso porque presenta un límite a las pedagogías que suponen a un otro como ausente o desde la racionalidad técnica, invitándolo a participar de un valor normalizado. Es necesario que la escuela diga que hay algo que no puede ser. No podemos ser esa niña o niño que nos está mirando mientras hablamos durante una clase. Hay otro ahí, el cual no soy yo. El yo que yo soy se encuentra con algo que no puede ser y, por eso, las diversidades invitan a escuchar, tal como desde otro marco de referencias invita el filósofo Han (2017): "resulta necesario volver a considerar la vida partiendo del otro, desde la relación con el otro, otorgándole al otro una prioridad ética, es más, aprendiendo de nuevo el lenguaje de la responsabilidad, escuchando y respondiendo al otro" (p. 110). Entonces, resulta necesario volver a pensar y reflexionar nuestras prácticas pedagógicas escuchando al otro en su ser-estar-en-el-mundo. 


\section{Consideraciones finales}

Hemos comenzado señalando que una apuesta para que las diversidades emerjan como proyecto por construirse requiere de una lectura que se vincule a diversas disciplinas, que uno de los desafíos para las diversidades puede ser la articulación de diferentes ámbitos del conocimiento. Abrirse a ello nos permite comprender que lo que está en disputa no son solo los saberes y acciones respecto de las diversidades o inclusión, sino las formas de ser y las formas de vida que están consideradas en ella. Para plantear una nueva forma de comprender las diversidades debemos volver a acordarnos que la educación tiene como fin y sentido la vida. Que la vida sea lo que pueda emerger significa que convivimos y coexistimos con otros mediante el maravilloso hecho de simplemente ser. Ese ser que habita en el lenguaje nos permite sentir y comprender el cuerpo, los silencios, nuestras relaciones con otros, nuestros dolores y alegrías, nuestras formas de conocer la naturaleza. Que emerja la vida significa que emerja a su vez un sentido ético, entendido como un habitar común y de pertenencia mutua. En ese sentido, la propuesta de Roger Slee (2012) tiene asidero: la inclusión se vuelve un proyecto político en la medida en que podemos vivir en un mundo común y no preestablecido en la ausencia del otro y en la invitación al otro a participar en el valor normalizado.

Las políticas de formación inicial docente en Chile, al definirse desde lo ajeno a lo común-acción, ajeno a la vida y al convivir, extravían al profesor en las posibilidades de reflexionar sobre las diversidades. Tanto así que adquiere sentido el epígrafe inicial de Roger Slee (2012): las escuelas son la experiencia del sufrimiento y la humillación. El sufrimiento de no poder ser nosotros mismos y la humillación de convertirnos en algo que no podemos definir. La ausencia de mecanismos que permitan ejercer el poder de preguntar, sentir y disentir sobre quiénes somos, en conjunto con otros, cierra a las escuelas a las diversidades. Seguiremos diciendo que el otro, el otro diferente desde la normalidad, es un ser deficiente (Skliar, 2002). Si somos capaces de abrir las puertas a otras pedagogías y a las diversidades, quizás también podremos abrir las puertas al ser y formas de apropiación del docente.

Es un aspecto esperanzador el hecho de que los docentes nos hayamos extraviado, porque nos impulsa a establecer nuevas preguntas que invitan a reconocer el ser docente desde la afectividad, la apertu- 
ra al mundo y la comprensión, considerando las diversidades como una forma de establecer lo común no como normalización, sino como escucha e interpretación. Quizás en la emergencia de una nueva forma de comprender al docente y a la escuela pueda desplegarse la discusión sobre cómo y para qué coexistir en un mundo diverso. La pregunta por el ser docente también es la pregunta por nuestra historia vital, por cómo queremos ser en lo común y lo diferente con los otros.

\section{Bibliografía}

Acevedo, J. (1999). Heidegger y la época de la técnica. Santiago, Chile: Editorial Universitaria.

Aranda, V. (2011). Reflexión y análisis de políticas y prácticas innovadoras a la luz de las representaciones sociales y de la necesidad de una educación intercultural en la formación inicial docente. Estudios Pedagógicos, 37(2), 301-314. DOI: http:// dx.doi.org/10.4067/S0718-07052011000200018.

Ávalos, B. (2013). ¿Héroes o Villanos? Profesión Docente en Chile. Santiago, Chile: Universitaria.

Bartolomé, L. (2008). La pedagogía crítica y la educación de los profesores y profesoras. Radicalización del profesorado futuro. En McLaren y Kincheloe (eds.), Pedagogía Crítica: dónde estamos, de qué hablamos. Barcelona: Editorial Grao.

Baker, B. (2002). The Hunt for Disability: The New Eugenics and the normalization of School Children. Teachers College Record, 104(4), 663-703.

Biesta, G. (2014). Measuring what we Value or Valuing what we Measure? Globalization, Accountability and the Question of Educational Purpose. Pensamiento Educativo. Revista de Investigación Educacional Latinoamericana, 51(1), 46-57. UC. DOI: 10.7764/PEL.51.1.2014.5

Casassus, J. (2006). La Educación del Ser Emocional. Santiago, Chile: Editorial Cuarto Propio.

Cherryholmes, C. (1999). Poder y Crítica: investigaciones post-estructurales en educación. Barcelona: Ediciones Pomares-Corredor.

Da Silva, T. T. (Org.). (2014) Identidade e diferenca: A perspectiva dos Estudos Culturais. Río de Janeiro: Petrópolis. 
De Gregorio, S. y Bruns, B. (2016). Práctica docente en el aula: una mirada al interior de la sala de clase en América Latina y el Caribe. En Manzi y García: Abriendo las puertas del Aula: transformación de las prácticas docentes. Santiago, Chile: Ediciones UC.

Druker, S. (2020). El giro epistemológico: De la diversidad de los otros a la diversidad como condición del encuentro. Revista de estudios y experiencias en educación, 19(39), 227-239. DOI: https://dx.doi.org/10.21703/rexe.20201939druker13

Duschatsky, S. (2017) Política de la escucha en la escuela. Buenos Aires: Paidós.

Fardella, C. y Sisto, V. (2015). Nuevas regulaciones del trabajo docente en Chile: discursos, subjetividad y resistencia. Psicología \& Sociedad, 27(1) 68-79. DOI: https://doi.org/10.1590/180703102015v27n1p068

Freire, P. (2016). El Maestro sin Recetas: el desafío de enseñar en un mundo cambiante. Buenos Aires: Editorial Siglo XXI.

Friz, C. (2016). La Universidad en disputa: sujeto, educación y formación universitaria en la concepción neoliberal. Santiago, Chile: Ceibo Ediciones.

Fuentealba, R. y Labra, P. (2011). Formación Inicial Docente: Una mirada al Proceso de Construcción de Conocimiento Profesional desde la Práctica. Foro Educacional, 19, 145-173. DOI: https://doi.org/10.29344/07180772.19.858

Garay, M., Sillar, M. (2018). Evaluación sobre los procesos formativos de los docentes en la región de Magallanes. Sophia Austral, (21), 27-42. DOI: https://dx.doi.org/10.4067/S071956052018000100027

Grundy, S. (1991). Producto o praxis del currículum. Madrid: Editorial Morata.

Guerra, P. y Balmaceda, C. (2015). Creencias epistemológicas en profesores que postulan al Programa de Acreditación de Excelencia Pedagógica: Análisis descriptivos y comparativos entre profesores que se desempeñan en los diferentes niveles de enseñanza. Estudios pedagógicos (Valdivia), 41(2), 107-125. DOI: https://dx.doi.org/10.4067/S0718-07052015000200007

Han, B. (2017). La expulsión de lo distinto. Barcelona. Editorial Herder. Heidegger, M. (1997). Ser y Tiempo. Santiago, Chile: Editorial Universitaria. 
Quay, J. (2013). Education, Experience and Existence Engaging Dewey, Peirce and Heidegger. Abingdon, Oxfordshire: Routledge.

López, V., González, P., Manghi, D., Ascorra, P., Oyanedel, J. C., Redón, S. y Salgado, M. (2018). Políticas de inclusión educativa en Chile: Tres nudos críticos. Archivos Analíticos de Políticas Educativas, 26(157). DOI: https://doi.org/http://dx.doi. org/10.14507/epaa.26.3088.

Manghi, D., Conejeros Solar, M.L., Bustos Ibarra, A., Aranda Godoy, I., Vega Córdova, V., Diaz Soto, K. y Gómez, A. L. (2020). Comprender la educación inclusiva chilena: Panorama de políticas e investigación educativa. Cadernos de Pesquisa, 50(175), 114-134. DOI: https://doi.org/10.1590/198053146605

Manzi, J. González, R. y Sun, Y. (2011). La Evaluación Docente en Chile. Santiago, Chile: Ediciones MIDEUC.

Masolo. D. A. (2014). Filosofía y conocimiento indígena: una perspectiva africana. En Boaventura de Sousa Santos y María Paula Meneses (eds.), Epistemologías del sur (perspectivas) (pp. 517-537). Madrid: Akal.

Matus, C. y Rojas, C. (2015). Normalidad y diferencia en nuestras escuelas: a propósito de la Ley de Inclusión Escolar. Revista Docencia, 20(56), 47-56.

Matus, C., Rojas, C., Guerrero, P., Herraz, P. C. y Sanyal, A. (2019). Diferencia y normalidad: producción etnográfica e intervención en escuelas. Revista Internacional de Investigación en Educación, 11(23), 23-38. DOI: 10.11144/Javeriana.m11-23.dnpe

Ministerio de Educación (2013). Música: Programa de estudio para Primer Año Básico. Unidad de Curriculum y Evaluación. Santiago, Chile.

Montecinos, C. (2016). Fortalecimiento del currículo de formación práctica en las carreras de pedagogía en Chile ¿Qué señala la evidencia empírica? En Manzi y García, Abriendo las puertas del Aula: transformación de las prácticas docentes. Santiago, Chile: Ediciones UC.

Montecinos, C. (2018). Vinculación Universidad Sistema Escolar: A través de y para la formación práctica. Ponencia en el marco del Seminario Internacional de Formación Inicial Docente y Aseguramiento de la Calidad en Chile, CNA. Recuperado de: https://www.cnachile.cl/Paginas/seminarios_y_congresos. aspx 
Muñoz, G. (2011). Docentes formados para la transformación del sistema educativo. Revista Docencia, (43), 76-85.

Naranjo, C. (2014). Cambiar la educación para cambiar el mundo. Santiago, Chile: Editorial Cuarto Propio-LOM.

Ocampo, A. (2018). Educación inclusiva: una teoría sin disciplina. Legados y recuperación de los saberes diaspóricos para una epistemología heterotópica. I Congreso Iberoamericano de Docentes, organizado por REDIB en conjunto con la Universidad de Cádiz, España. REDIB-Universidad de Cádiz, Algeciras. Recuperado de: https://www.aacademica.org/aldo. ocampo.gonzalez $/ 3$

Osandón, L., Caro, M., Magendzo, A., Lavín, S., González, F. y Cabaluz, J. (2018). Estado, mercado y currículum escolar: La experiencia chilena (1964-2018); Cuestiones fundamentales y actuales del currículo, el aprendizaje y la evaluación. OIEUNESCO. DOI: 10.13140/RG.2.2.15634.35522

Parcerisa, L. y Falabella, A. (2017). La consolidación del Estado evaluador a través de políticas de rendición de cuentas: Trayectoria, producción y tensiones en el sistema educativo chileno. Education Policy Analysis Archives, 25(89). DOI: http:// dx.doi.org/10.14507/epaa.25.3177

Parra Muñoz, H., Garrido Reyes, C., Carrasco Lobos, A., Vergara Rodríguez, M., Hidalgo Kawada, F. y Meza Buzzetti, D. (2020). Normocentrismo, diversidad y alteridades: Deconstruyendo las políticas educativas inclusivas en Chile. Archivos Analíticos de Políticas Educativas, 28(100). DOI: https://doi. org/10.14507/epaa.28.4338

Restrepo-Pérez, O. E., Restrepo-Pérez, L. M. R. y Jaramillo-Ocampo, D. A. J. (2017). La formación: Una apuesta por las pedagogías del encuentro. Revista Latinoamericana de Estudios Educativos, 13(2). Recuperado de: https://www.redalyc.org/articulo.oa?id=134154501006.

Rancière, J. (2007). El maestro ignorante: cinco lecciones sobre la emancipación intelectual. Buenos Aires: Libros del Zorzal.

Recalcati, M. (2016). La hora de clases: por una erótica de la enseñanza. Barcelona: Anagrama.

Romanowski, M. (1997). Teachers' Lives and Beliefs: Influences That Shape the U. S. History Curriculum. Chicago, IL: Annual Meeting of the American Educational Research Association. 
Skliar, C (2002) ¿Y si el otro no estuviera ahí? Notas para una pedagogía improbable de la diferencia. Buenos Aires: Artes Gráficas.

Skliar, C. (2005). Poner en tela de juicio la normalidad, no la anormalidad. Políticas y falta de políticas en relación con las diferencias en educación. Revista Educación y Pedagogía, 17(41), 11-22. Recuperado de: https://revistas.udea.edu.co/index. $\mathrm{php} /$ revistaeyp/article/view/6024.

Skliar, C. (2016). Preguntar la diferencia: cuestiones sobre la inclusión. Sophia, 11(1), 33-43. Recuperado de: https://revistas. ugca.edu.co/index.php/sophia/article/view/297

Slee, R. (2012). La escuela extraordinaria: exclusión, escolarización y educación inclusiva. Madrid: Morata.

Sverdlick, I. (2019). Inclusión educativa y derecho a la educación. La disputa de los sentidos. Education Policy Analysis Archives, 27(26), 1-24. DOI: https://doi.org/10.14507/epaa.27.3197

Tardif, M. (2004). Los saberes del docente y su desarrollo profesional. Madrid: Narcea.

Uribe, L. (2002). Hermenéutica, posmodernidad y ética: aproximaciones a partir de Gianni Vattimo. Revista Chilena de temas sociológicos, 41-55. Recuperado de: http://ediciones.ucsh.cl/ ojs/index.php/TSUCSH/article/view/179

Welsh, W. (2008). Heidegger: antropocentrismo ontológico. En Heidegger: sendas que vienen. Vol. 1 (pp. 87-113). Madrid: UA Ediciones. 